\title{
Design, Synthesis and Gelation of Low Molecular Weight Organo-gelators derived from Oleic acid via, amidation
}

\author{
NARENDRA S. JOSHI ${ }^{1}$, GOVINDA P. WAGHULDE ${ }^{2}$, \\ GAURAV R. GUPTA ${ }^{3 *}$ and RAJU P. PHALAK ${ }^{2}$ \\ ${ }^{1}$ Department of Chemistry, Arts, Commerce \& Science College, Bodwad-425310, Maharashtra, India. \\ 2Department of Chemistry, D.D.N. Bhole College, Bhusawal-425201, Maharashtra, India. \\ ${ }^{3}$ Department of Chemistry, GTP College, Nandurbar-425412, Maharashtra, India. \\ ${ }^{*}$ Corresponding author E-mail: grgupta83@gmail.com \\ http://dx.doi.org/10.13005/ojc/370514 \\ (Received: July 27, 2021; Accepted: September 12, 2021)

\begin{abstract}
In recent decades, gels have been widely considered for various medicinal purposes and, in particular, wound healing applications. In this regard, amides of oleic acids and 9, 10-dihydroxyoctadecanoic acid are synthesized and characterized with the help of modern analytical tools. Among the mentioned amide frameworks, $\mathrm{N}$-(2-aminoethyl)-oleamide exhibits high order of gelation not only with different organic solvents such as $n$-hexane and DMSO but also with different edible oils such as sesame oil, mustard oil, coconut oil and citriodora oil. Here, we briefly discuss the optimization of gelation conditions for the synthesized amides as organo-gelator, in addition to that the minimum gelation concentration and gelation temperature have also been studied.
\end{abstract}

Keywords: Organo-gelator, Minimum gelation concentration, Gelation temperature, Amidation.

\section{INTRODUCTION}

Gels are unique materials. They have been known since ancient times such that their origin can be found at least Neolithic times and, ever since, they have played an important role in many decisive advances of technology, art and medicine. The properties of the gel largely depend on the structure of the polymeric network formed and the interaction of the solute with the solvent. The external stimuli such as $\mathrm{pH}$, temperature, light and electrical fields have significant impact on the gelation and the nature of the gel.
However, the stimulation has been depending on the nature of solute or a gelator. ${ }^{1-2}$ Most of the gelators have very complex arrangements of the atoms although a few are quite simple molecules has both lyophilic and hydrophobic or polar regions and functional groups that offers hydrogen bonding interactions. $^{3-6}$ In addition to that, a broad range of organic liquids have already been gelled, including quasi-liquids such as supercritical $\mathrm{CO}_{2}$ and water as well. ${ }^{7-8}$ The characteristic structure induced and diffusional properties of organo-gels have a broad range of application window in modern disciplines of the colloidal science. ${ }^{9-20}$ In this regard, variety of

This is an Open Access article licensed under a Creative Commons license: Attribution 4.0 International (CC- BY). Published by Oriental Scientific Publishing Company @ 2018

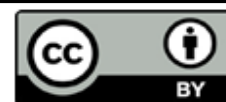


organic frameworks have already been exploited as a gelator to achieve the gel of desired properties. Amongst these organic frameworks nitrogen containing organic compounds such as primary and secondary amide like urea, carbamates, thioureas, and other classes which have strong tendency to form $\mathrm{H}$-bond interactions shows promising results. ${ }^{3}$ One class of chemical compounds that is present as a building block in all living systems are so-called amides. ${ }^{21-22}$ Even though their significance is apparent, they have often been unnoticed as low molecular weight organo-gelators in the science of molecules. Indeed, an exceptional stability of the amide frameworks, an assurance of their abundant presence in life governing processes, is manifested their inertness to engage with common organic solvents or reagents. Intermolecular hydrogen bonding between the amide functional groups provides the driving force for the formation of extended aggregates in nonpolar, organic solvents, whereas the alkyl chains are thought to ensure compatibility with nonpolar solvents to increase solubility and prevent crystallization ${ }^{23-24}$.

Organogels of edible oils have attracted the attention in food science as an alternative to the hydrogenation of oils, which is related to the development of cardiovascular diseases. Gelation can be used for structuring of oils. The gelation of oils depends on the structural properties of gelator as well as the oil. The type of oil and hydrophilic head of the gelator exert influence on the physical properties of the gels ${ }^{25-27}$.

In this work, we aimed to synthesize nitrogen containing derivatives of oleic acid, by using usual methods available in the literature by making suitable changes eventually, and study gelation of several organic liquids by using them. The oleic acid is selected because it has a long chain of carbon atoms and acid functional group, which can be converted into amide easily. The long chain of carbon atoms is able to form van der Waals forces and the amide group can form hydrogen bonds. For this purpose, we synthesized eight different derives for this investigation. It is observed that, the gelator, $\mathrm{N}$-(2-aminoethyl)-oleamide (2), is very efficient towards the gelation of edible oils as compared to the organic liquids.

\section{EXPERIMENTAL}

\section{Materials}

Oleic acid, ammonia solution, potassium permanganate, sodium hydroxide (pellets), hydrochloric acid, ethanol, activated charcoal was purchased from Loba Chemie. Thionyl chloride, ethylene diamine, phenyl hydrazine, p-toluidine, ethyl acetate was purchased from Fisher Scientific. Edible oils are purchased from local market. All other solvents (extra pure), used for gelation, are purchased from Fisher Scientific. Melting points were confirmed using DSC.

\section{FT-IR analysis}

Fourier Transform Infrared (FTIR) analysis of the synthesized ILs was performed on the FTIR affinity model, Shimadzu; dry $\mathrm{KBr}$ was used to set the background; thereafter, a $\mathrm{KBr}$ pellet was developed and analysed. Prior to the analysis, the instrument was calibrated by performing an IR scan of a polystyrene film.

\section{NMR $\left({ }^{1} \mathrm{H}\right.$ and $\left.{ }^{13} \mathrm{C}\right)$ analysis}

${ }^{1} \mathrm{H}-\mathrm{NMR}$ and ${ }^{13} \mathrm{C}-\mathrm{NMR}$ spectra are recorded on Bruker Avance Neo $500 \mathrm{MHz}$ NMR spectrometer at SAIF, Punjab University, Chandigarh. Tetramethylsilane (TMS) is used as internal standard to measure the chemical shift values in ppm and $\mathrm{CDCl}_{3}$ is used as solvent.

\section{Mass Spectral analysis}

Mass Spectra are recorded on Waters Q-TOF Micromass (ESI-MS) Spectrometer at SAIF, Punjab University, Chandigarh.

\section{TGA-DSC analysis}

The TGA-DSC analysis of the gels was performed at Shivaji University, Kolhapur, on the TA instrument (model: SDT Q $600 \mathrm{~V}$ 20.9). Further details about the experimentation has already been reported in our previous communications. ${ }^{29-33}$

\section{General procedure for the amidation of oleic acid}

A round bottom flask, fitted with water condenser was charged with $20 \mathrm{~mL}$ of thionyl chloride and $52 \mathrm{~mL}$ of oleic acid was added very slowly through condenser [Please note that rapid addition can cause bumping or explosion] with constant stirring into it. After complete addition of oleic acid, the reaction mixture was allowed to 
heat at $60^{\circ} \mathrm{C}$ for $15 \mathrm{~min}$ with continuous stirring. The progress of the reaction was monitored using TLC. After completion of the reaction, the reaction mixture was allowed to cool in an ice- bath and the unreacted acid was confirmed by treating the reaction mass with $10 \%$ sodium bicarbonate solution. Thereafter, various amines namely ammonia, ethylene diamine, phenyl hydrazine and $\mathrm{m}$-anisidine ( $\mathrm{m}$-anisidine was taken into acetone) respectively were added to this ice-cold solution to obtain corresponding amides of oleic acid in good to excellent yield. The crystals of the crude product were separated out as a white solid (oleamide), white solid (N-(2-aminoethyl)-oleamide), yellowish solid ((Z)-N'-phenyloleohydrazide), and pink solid ((Z)-N-(3-methoxyphenyl)oleamide), and were collected over suction pump and stored in a desiccator Scheme 1.

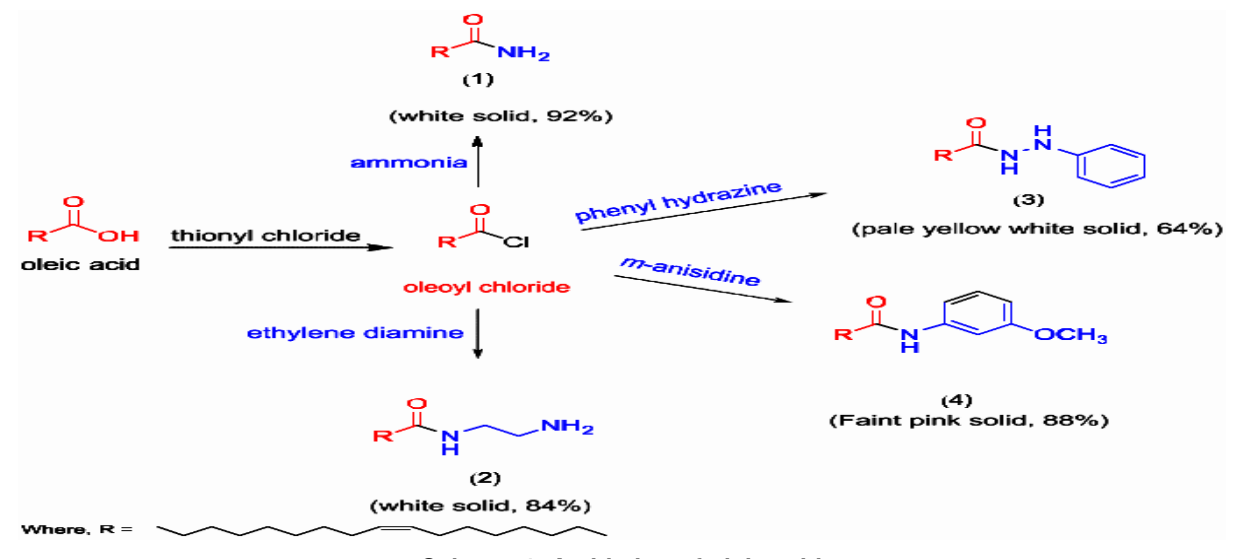

Scheme 1. Amidation of oleic acid

General procedure for the synthesis of $\mathbf{9}$, into it. After complete addition of an acid, the 10-dihydroxyoctadecanoic acid (5)

An aqueous solution of $1 \mathrm{~mL}$ of oleic acid and $1 \mathrm{~g}$ sodium hydroxide was allowed to cool in an ice bath. Thereafter, an aqueous solution of $0.80 \mathrm{~g}$ of potassium permanganate was added to this solution very slowly. Then, $30 \mathrm{~mL}$ of hydrochloric acid was added with occasional stirring to the reaction mixture. A brown solid was formed within 30 min which was collected over suction pump and recrystallized from ethanol Scheme 2.

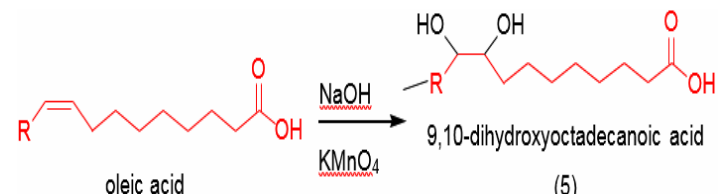

oleic acid

(5)

(White solid, 84\%)

Scheme 2. Hydroxylation of oleic acid

General procedure for amidation of $\mathbf{9}$, 10-dihydroxyoctadecanoic acid

A round bottom flask, fitted with water condenser was charged with $20 \mathrm{~mL}$ of thionyl chloride and a solution of 5 of 9,10-dihydroxyoctadecanoic acid in acetone was added very slowly through condenser [Please note that rapid addition can cause bumping or explosion] with constant stirring reaction mixture was allowed to heat at $60^{\circ} \mathrm{C}$ for 15 min with continuous stirring. The progress of the reaction was monitored using TLC. After completion of the reaction, the reaction mixture was allowed to cool in an ice-bath and the presence of unreacted acid was confirmed by treating the reaction mass with $10 \%$ sodium bicarbonate solution. Thereafter, various amines namely ammonia and ethylene diamine respectively were added to this ice-cold solution to obtained corresponding amides of 9,10-dihydroxyoctadecanoic acid in measurable yields. The crystals of the crude product were separates-out as a waxy white solid for corresponding amides (9,10-diaminooctadecanamide and 9 , 10-bis(2-aminoethylamino)-N-(2-aminoethyl) octadecaneamide, respectively) was collected over suction pump and stored in a desiccator (Scheme 3).

\section{Gelation experiments}

Finally gelation using synthesized organogelators was performed by heating the mixture of a small, weighed quantity of gelator in appropriate solvent, up to the boiling point of solvent. Gel formation was ensured by inverted tube method. 


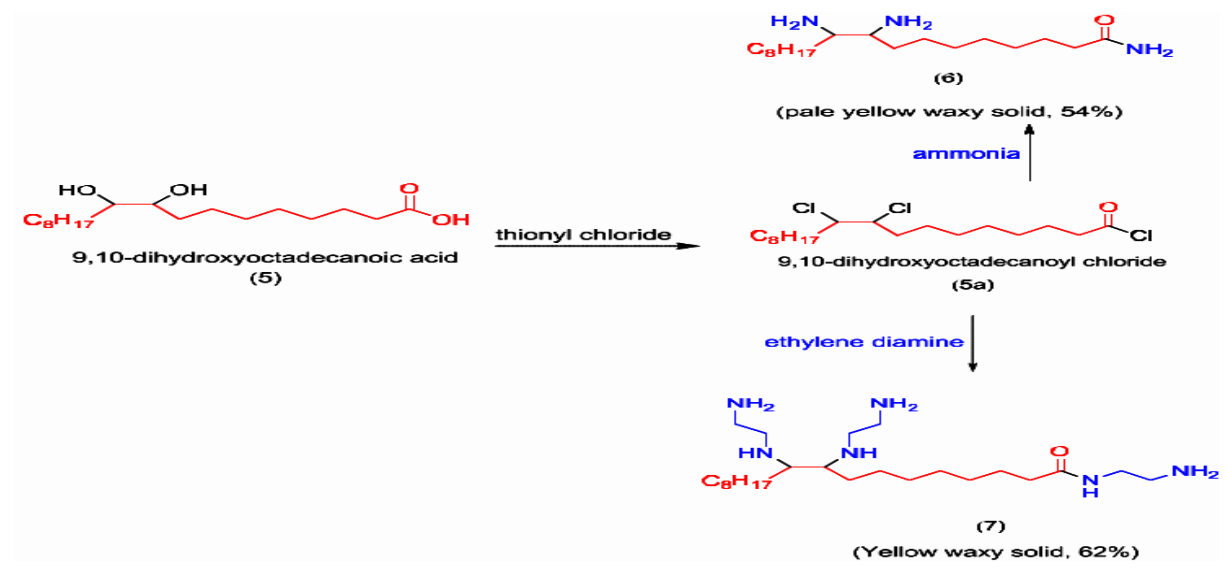

Scheme 3. Amidation of 9,10-dihydroxyoctadecanoic acid

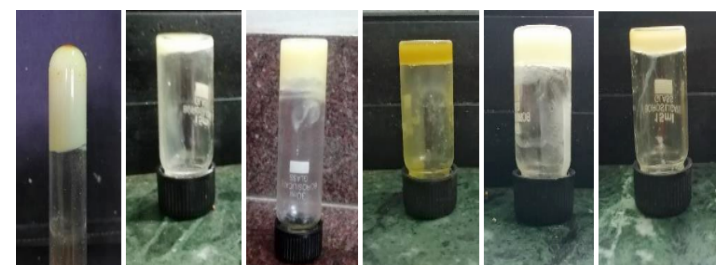

Fig. 1. Gels of n-Hexane, DMSO, sesame oil, mustard oil, coconut oil, citriodora oil

\section{RESULTS AND DISCUSSION}

After successful amidation of oleic acid and 9,10-dihydroxyoctadecanoic acid, the resulting amides ( 1 to 7 ) were fully analysed by means of modern spectroscopic techniques, and the resulting spectral data of all the synthesized derivatives is given below.

\section{Oleamide (1)}

FT-IR: $1645.6 \mathrm{~cm}^{-1}$ (N-H bending), 2618.5 $\mathrm{cm}^{-1}$ and $2819.7 \mathrm{~cm}^{-1}$ (aliphatic $\mathrm{C}-\mathrm{H}$ stretch), 807.0 $\mathrm{cm}^{-1}$ (C=C stretch), $3021.0 \mathrm{~cm}^{-1}$ (alkene $\mathrm{C}-\mathrm{H}$ stretch), 1444.3 (C-N stretch); ${ }^{1} \mathrm{H}-\mathrm{NMR}\left(500 \mathrm{MHz}, \mathrm{CDCl}_{3}\right)$ : $\delta 0.87\left(\mathrm{t}, 3 \mathrm{H}, \mathrm{CH}_{3}\right), \delta 1.25-1.61\left(\mathrm{~m}, 22 \mathrm{H}, \mathrm{CH}_{2}\right), \delta$ 1.98-2.03 (m, 4H, $\left.\mathrm{CH}_{2}\right), \delta 2.30\left(\mathrm{t}, 2 \mathrm{H}, \mathrm{CH}_{2}\right), \delta 5.30-$ $5.37(\mathrm{~m}, 2 \mathrm{H}, \mathrm{HC}=\mathrm{CH}), \delta 5.83(\mathrm{~s}, 2 \mathrm{H}, \mathrm{NH}) ;{ }^{13} \mathrm{C}-\mathrm{NMR}$ $\left(500 \mathrm{MHz}, \mathrm{CDCl}_{3}\right)$ : 14.04, 22.55, 24.87, 25.50, 27.20, 29.30, 31.50, 31.88, 32.58, 34.28, 35.96, 129.73, 177.16; Analytically calculated for $\mathrm{C}_{18} \mathrm{H}_{35} \mathrm{NO} . \mathrm{m} / \mathrm{z}$ : 281.35 ; m.p.: $94.54^{\circ} \mathrm{C}$.

\section{N-(2-aminoethyl)-oleamide (2)}

FT-IR: $3300.6 \mathrm{~cm}^{-1}$ (N-H stretch), 1640.0 $\mathrm{cm}^{-1}$ (Amide $\mathrm{C}=\mathrm{O}$ stretch), $2853.3 \mathrm{~cm}^{-1}$ and 2920.4 $\mathrm{cm}^{-1}$ (aliphatic $\mathrm{C}-\mathrm{H}$ stretch), $717.5 \mathrm{~cm}^{-1}(\mathrm{C}=\mathrm{C}$ stretch), $3088.1 \mathrm{~cm}^{-1}$ (alkene $\mathrm{C}-\mathrm{H}$ stretch); ${ }^{1} \mathrm{H}-\mathrm{NMR}(500$ $\left.\mathrm{MHz}, \mathrm{CDCl}_{3}\right): \delta$ 0.86-0.89 (t, $\left.3 \mathrm{H}, \mathrm{CH}_{3}\right), \delta 1.25-1.36$ (m, 22H, $\left.\mathrm{CH}_{2}\right), \delta 1.59-1.61\left(\mathrm{~m}, 4 \mathrm{H}, \mathrm{CH}_{2}\right), \delta 1.96-2.05$ (m, 4H, CH2), $\delta$ 2.15-2.16 (t, 2H, $\left.\mathrm{CH}_{2}\right), \delta$ 3.37-3.38 (t, 2H, $\mathrm{NH}_{2}$ ), $\delta 5.30-5.37(\mathrm{~m}, 2 \mathrm{H}, \mathrm{HC}=\mathrm{CH}), \delta 6.28$ (s, $1 \mathrm{H}, \mathrm{NH}) ;{ }^{13} \mathrm{C}-\mathrm{NMR}\left(500 \mathrm{MHz}, \mathrm{CDCl}_{3}\right): 14.07$, 22.57, 22.68, 25.73, 27.18, 29.17, 29.31, 29.33, 29.37, 29.53, 29.67, 29.71, 29.73, 31.52, 31.92, $36.70,40.18,127.90,130.01,174.54$; Analytically calculated for $\mathrm{C}_{20} \mathrm{H}_{40} \mathrm{~N}_{2} \mathrm{O} \cdot \mathrm{m} / \mathrm{z}: 324(\mathrm{M}+1325.42$ and $\mathrm{M}+2$ 326.19): m.p.:93.91 ${ }^{\circ} \mathrm{C}$.

\section{(Z)-N'-phenyloleohydrazide (3)}

FT-IR: $3205.5 \mathrm{~cm}^{-1}$ (N-H stretch), 1589.7 $\left(\mathrm{N}-\mathrm{H}\right.$ bend), $1640.0 \mathrm{~cm}^{-1}$ (Amide $\mathrm{C}=\mathrm{O}$ stretch), $2931.6 \mathrm{~cm}^{-1}$ and $2998.6 \mathrm{~cm}^{-1}$ (aliphatic $\mathrm{C}-\mathrm{H}$ stretch), $762.2-678.4 \mathrm{~cm}^{-1}$ (C-H bend), $1494.7 \mathrm{~cm}^{-1}$ (C-C ring stretch), $885.2 \mathrm{~cm}^{-1}$ ( $\mathrm{C}=\mathrm{C}$ bend), $3088.1 \mathrm{~cm}^{-1}$ (alkene C-H stretch), 1231.9 (C-N stretch); ${ }^{1} \mathrm{H}-\mathrm{NMR}$ (500 $\left.\mathrm{MHz}, \mathrm{D}_{2} \mathrm{O}\right), \delta 0.99\left(\mathrm{t}, 3 \mathrm{H}, \mathrm{CH}_{3}\right), \delta$ 1.01-1.08 (m, $\left.18 \mathrm{H}, \mathrm{CH}_{2}\right), \delta 1.34\left(\mathrm{~m}, 4 \mathrm{H}, \mathrm{CH}_{2}\right), \delta 1.88-1.90(\mathrm{~m}, 4 \mathrm{H}$, $\left.\mathrm{CH}_{2}\right), \delta 2.12\left(\mathrm{t}, 2 \mathrm{H}, \mathrm{CH}_{2}\right), \delta 3.45-3.49(\mathrm{~d}, 1 \mathrm{H}, \mathrm{NH})$, $\delta$ 3.91-3.95 (d, 1H, NH), $\delta 5.09(\mathrm{~m}, 2 \mathrm{H}, \mathrm{HC}=\mathrm{CH})$,

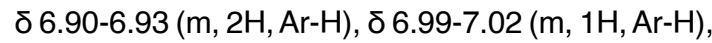
ठ 7.25-7.28 (m, 2H, Ar-H); ${ }^{13} \mathrm{C}-\mathrm{NMR}\left(500 \mathrm{MHz}, \mathrm{D}_{2} \mathrm{O}\right)$, 13.24, 16.81, 20.42, 20.52, 57.41, 61.69, 115.55, 123.80, 129.69,129.91, 143.38, 174.59, 176.57; Analytically calculated for $\mathrm{C}_{24} \mathrm{H}_{4} 0 \mathrm{~N}_{2} \mathrm{O} \cdot \mathrm{m} / \mathrm{z}: 374$ $(\mathrm{M}+2):$ m.p.: $156.98^{\circ} \mathrm{C}$.

\section{(Z)-N-(3-methoxyphenyl)oleamide (4)}

FT-IR: $2830.9 \mathrm{~cm}^{-1}$ and $2562.5 \mathrm{~cm}^{-1}$ (aliphatic C-H stretch), $851.7-723.1 \mathrm{~cm}^{-1}$ ( $\mathrm{C}-\mathrm{H}$ bend), $1600.9-1545.0 \mathrm{~cm}^{-1}$ (C-C ring stretch), $924.4 \mathrm{~cm}^{-1}$ ( $\mathrm{C}=\mathrm{C}$ bend), $1640 \mathrm{~cm}^{-1}$ ( $\mathrm{C}=\mathrm{O}$ stretch), 1265.4 (C-N stretch); ${ }^{1} \mathrm{H}-\mathrm{NMR}\left(500 \mathrm{MHz}, \mathrm{CDCl}_{3}\right): \delta$ 0.86-0.89 $\left(\mathrm{t}, 3 \mathrm{H}, \mathrm{CH}_{3}\right), \delta 1.25-1.31\left(\mathrm{~m}, 22 \mathrm{H}, \mathrm{CH}_{2}\right), \delta 1.60-1.65$ $\left(\mathrm{m}, 2 \mathrm{H}, \mathrm{CH}_{2}\right), \delta$ 1.94-2.02 (m, 2H, $\left.\mathrm{CH}_{2}\right), \delta 2.32-2.35$ 
$\left(\mathrm{m}, 2 \mathrm{H}, \mathrm{CH}_{2}\right), \delta 3.70\left(\mathrm{~s}, 3 \mathrm{H}, \mathrm{OCH}_{3}\right), \delta 7.72(\mathrm{~s}, 1 \mathrm{H}$, $\mathrm{NH}), \delta$ 5.32-5.38 (m, 2H, HC=CH), $\delta 7.26(\mathrm{~m}, 1 \mathrm{H}$, Ar-H), $\delta 7.45-7.48(\mathrm{~m}, 1 \mathrm{H}, \mathrm{Ar}-\mathrm{H}), \delta 7.55-7.58(\mathrm{~m}$, $1 \mathrm{H}, \mathrm{Ar}-\mathrm{H}), \delta$ 7.95-7.98 (m, 1H, Ar-H); ${ }^{13} \mathrm{C}-N M R(500$ $\left.\mathrm{MHz}, \mathrm{CDCl}_{3}\right): 26.61,27.22,28.96,29.25,29.68$, 29.77, 31.91, 32.60, 33.84, 128.32, 130.03, 130.50, 133.12, 178.64, 198.26; Analytically calculated for $\mathrm{C}_{25} \mathrm{H}_{41} \mathrm{NO}_{2}$. m/z:387; m.p.: $172.75^{\circ} \mathrm{C}$.

\section{9,10-dihydroxyoctadecanoic acid (5)}

FT-IR: $3272.6 \mathrm{~cm}^{-1}$ (O-H stretch, alcohol), $3188.7 \mathrm{~cm}^{-1}$ (O-H stretch, carboxylic acid), 2914.8 $\mathrm{cm}^{-1}$ and $2847.7 \mathrm{~cm}^{-1}$ (aliphatic $\mathrm{C}-\mathrm{H}$ stretch), 1069.7 $\mathrm{cm}^{-1}$ ( C-O stretch, secondary alcohol), $1695.9 \mathrm{~cm}^{-1}$ $\left(\mathrm{C}=\mathrm{O}\right.$ stretch, carboxylic acid), $1433.2 \mathrm{~cm}^{-1}(\mathrm{C}=\mathrm{O}$ bend, carboxylic acid); ${ }^{1} \mathrm{H}-\mathrm{NMR}\left(500 \mathrm{MHz}, \mathrm{CDCl}_{3}\right)$ : $\delta$ 0.86-0.89 (t, 3H, $\left.\mathrm{CH}_{3}\right), \delta 1.25-1.30\left(\mathrm{~m}, 26 \mathrm{H}, \mathrm{CH}_{2}\right)$, $\delta 2.19-2.21\left(\mathrm{~m}, 2 \mathrm{H}, \mathrm{CH}_{2}\right), \delta 2.22-2.28\left(\mathrm{~m}, 2 \mathrm{H}, \mathrm{CH}_{2}\right)$,

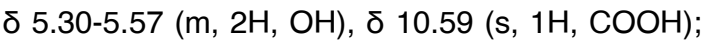
${ }^{13} \mathrm{C}-\mathrm{NMR}\left(500 \mathrm{MHz}, \mathrm{CDCl}_{3}\right): 14.10,22.68,24.70$, 27.22, 28.62, 29.14, 29.24, 29.43, 29.52, 29.68, $31.90,34.07,129.73$; Analytically calculated for $\mathrm{C}_{18} \mathrm{H}_{36} \mathrm{O}_{4}$. m/z: 316; m.p.; $122.29^{\circ} \mathrm{C}$.

\section{9, 10-diaminooctadecanamide (6)}

FT-IR: $1651.2 \mathrm{~cm}^{-1}$ (C=O stretch, primary amide), $3390.0 \mathrm{~cm}^{-1}$ (N-H stretch, amine), 1198.3 $\mathrm{cm}^{-1}$ (C-N stretch, amine), $2920.4 \mathrm{~cm}^{-1}$ and 2853.3 $\mathrm{cm}^{-1}$ (aliphatic $\mathrm{C}-\mathrm{H}$ stretch); ${ }^{1} \mathrm{H}-\mathrm{NMR}(500 \mathrm{MHz}$, $\left.\mathrm{CDCl}_{3}\right): \delta 0.88\left(\mathrm{t}, 3 \mathrm{H}, \mathrm{CH}_{3}\right), \delta 1.25-1.29(\mathrm{~m}, 2 \mathrm{H}$, $\left.\mathrm{CH}_{2}\right), \delta 1.61\left(\mathrm{~m}, 4 \mathrm{H}, \mathrm{CH}_{2}\right), \delta 2.00-2.01\left(\mathrm{~m}, 2 \mathrm{H}, \mathrm{CH}_{2}\right)$, $\delta$ 2.15-2.19 (m, 2H, $\left.\mathrm{CH}_{2}\right), \delta 2.86-2.95(\mathrm{~m}, 2 \mathrm{H}, \mathrm{CH})$, $\delta 5.19-5.37\left(\mathrm{~d}, 4 \mathrm{H}, \mathrm{NH}_{2}\right), \delta 6.34\left(\mathrm{~s}, 2 \mathrm{H}, \mathrm{CONH}_{2}\right)$; ${ }^{13} \mathrm{C}-\mathrm{NMR}\left(500 \mathrm{MHz}, \mathrm{CDCl}_{3}\right): 14.10,27.22,29.09$, 29.28, 29.35, 29.51, 29.58, 29.76, 31.26, 34.35, $36.78,41.17,60.19,74.68,77.23,129.74,129.99$, 173.88; Analytically calculated for $\mathrm{C}_{18} \mathrm{H}_{39} \mathrm{~N}_{3} \mathrm{O} . \mathrm{m} / \mathrm{z}$ : 313; m.p.; $63.64^{\circ} \mathrm{C}$.

\section{9, 10-bis(2-aminoethylamino)- $\mathrm{N}$-(2-aminoethyl) octadecaneamide (7)}

FT-IR: $1640.0 \mathrm{~cm}^{-1}(\mathrm{C}=\mathrm{O}$ stretch, secondary amide), $3283.8 \mathrm{~cm}^{-1}\left(\mathrm{~N}-\mathrm{H}\right.$ stretch, amine), $1550 \mathrm{~cm}^{-1}$ ( $\mathrm{N}-\mathrm{H}$ bend, amine), $1159.2 \mathrm{~cm}^{-1}$ (C-N stretch, amine), $2920.4 \mathrm{~cm}^{-1}$ and $2853.3 \mathrm{~cm}^{-1}$ (aliphatic $\mathrm{C}-\mathrm{H}$ stretch); ${ }^{1} \mathrm{H}-\mathrm{NMR}\left(500 \mathrm{MHz}, \mathrm{CDCl}_{3}\right): \delta 0.88\left(\mathrm{t}, 3 \mathrm{H}, \mathrm{CH}_{3}\right), \delta$ $1.25\left(\mathrm{~m}, 18 \mathrm{H}, \mathrm{CH}_{2}\right), \delta 1.77-1.78\left(\mathrm{~m}, 8 \mathrm{H}, \mathrm{CH}_{2}\right), \delta$ $2.16\left(\mathrm{~m}, 2 \mathrm{H}, \mathrm{CH}_{2}\right), \delta 2.28\left(\mathrm{~m}, 2 \mathrm{H}, \mathrm{CH}_{2}\right), \delta 2.37-2.39$ (m, 4H, $\left.\mathrm{CH}_{2}\right), \delta 2.87\left(\mathrm{~m}, 4 \mathrm{H}, \mathrm{CH}_{2}\right), \delta 3.37-3.41(\mathrm{~m}$, $\left.2 \mathrm{H}, \mathrm{CH}_{2}\right), \delta 3.59\left(\mathrm{~m}, 2 \mathrm{H}, \mathrm{CH}_{2}\right), \delta 4.11\left(\mathrm{t}, 6 \mathrm{H}, \mathrm{NH}_{2}\right)$, $\delta 4.12(\mathrm{~m}, 2 \mathrm{H}, \mathrm{NH}), \delta 8.04(\mathrm{t}, 1 \mathrm{H}, \mathrm{CONH}) ;{ }^{13} \mathrm{C}-\mathrm{NMR}$ $\left(500 \mathrm{MHz}, \mathrm{CDCl}_{3}\right): 14.10,29.03,29.16,29.31,29.43$, $29.55,29.69,31.16,31.82,31.89,32.60,34.39$, $39.14,40.11,60.15,60.18,65.61,74.67,74.74$, $77.03,129.71,129.75,129.99,173.93$; Analytically calculated for $\mathrm{C}_{24} \mathrm{H}_{54} \mathrm{~N}_{6} \mathrm{O}$. m/z: 442 ; M.P.; $79.93^{\circ} \mathrm{C}$.

Once their structure and purity has been confirmed these amides were utilised as an organo-gelators towards the gelation of organic solvents (Table 1 , entry $a-p$ ) and oils (Table 1 , entry q-v) as well.

The closer scrutiny of Table 1 , reveals that all the gelators- 1 to 7 are insoluble in water (entry, a) and having high solubility in $\mathrm{CCl}_{4}$ (entry, c), chloroform (entry, d), acetone (entry, e), toluene (entry, i), methanol (entry, j), ethanol (entry, k), o-xylene (entry, n), p-xylene (entry, o) and chlorobenzene (entry, p). Furthermore, gelator- 1 is found insoluble in acetophenone (entry, h), 1-butanol (entry, I), silicone oil (entry, m), sesame oil (entry, q), mustard oil (entry, r), eucalyptus oil (entry, s), coconut oil (entry, t), cinnamon oil (entry, $u$ ), and citriodora oil (entry, v). However, in DMSO gelator-1 forming a precipitate. In case of gelator-3, the experimental data shows that the gelator-3 have very low or partial solubility in nitrobenzene (entry, g), silicone oil (entry, m), sesame oil (entry, q), mustard oil (entry, r), and coconut oil (entry, t), and very interestingly no precipitate formation have been encountered in case of gelator-3. Next, in case of gelator-4, the experimental data clears that gelator- 4 is found to be insoluble in nitrobenzene (entry, g), mustard oil (entry, r), eucalyptus oil (entry, s), coconut oil (entry, t), and cinnamon oil (entry, u), while it has an ability to form a precipitate with sesame oil (entry, q). In case of gelator-5, either it is soluble (entry, $b-p)$ or it is insoluble (entry, $a$, and $q-v)$. In the next, gelator- 6 and gelator-7 exhibits very similar response with all the studied organic solvents and with oils except with citriodora oil (entry, v). To our delight, the experimental data also evidenced that gelator-2 (N-(2-aminoethyl)-oleamide), exhibits an exceptional behaviour with the organic solvents as well as with an oils. gelator-2 (N-(2-aminoethyl)-oleamide), results an opaque gel with $n$-hexane (entry, b), DMSO (entry, f), sesame oil (entry, q), mustard oil (entry, r), coconut oil (entry, t), and with citriodora oil (entry, v). 
Table 1: Gelation data obtained with all the synthesized gelators-1 to 7 at $2 \mathrm{wt} \% / \mathrm{v}$ concentration in various organic solvents and oils

\begin{tabular}{|c|c|c|c|c|c|c|c|c|}
\hline Entry & Gelators solvents & 1 & 2 & 3 & 4 & 5 & 6 & 7 \\
\hline a & Water & 1 & I & 1 & I & I & 1 & I \\
\hline$b$ & $\underline{n}$-Hexane & $\mathrm{S}$ & OG & $S$ & $\mathrm{~S}$ & $\mathrm{~S}$ & $\mathrm{~S}$ & S \\
\hline $\mathrm{C}$ & $\mathrm{CCl}_{4}$ & $\mathrm{~S}$ & $S$ & $\mathrm{~S}$ & $\mathrm{~S}$ & $\mathrm{~S}$ & $\mathrm{~S}$ & S \\
\hline$d$ & Chloroform & $\mathrm{S}$ & $S$ & $\mathrm{~S}$ & $\mathrm{~S}$ & $\mathrm{~S}$ & $\mathrm{~S}$ & S \\
\hline e & Acetone & $\mathrm{S}$ & $S$ & $\mathrm{~S}$ & $\mathrm{~S}$ & $\mathrm{~S}$ & $\mathrm{~S}$ & $S$ \\
\hline$f$ & DMSO & $\mathrm{P}$ & $O G$ & $\mathrm{~S}$ & $\mathrm{P}$ & $\mathrm{S}$ & I & I \\
\hline $\mathrm{g}$ & Nitrobenzene & $S$ & $\mathrm{~S}$ & 1 & 1 & $\mathrm{~S}$ & I & I \\
\hline $\mathrm{h}$ & Acetophenone & 1 & $\mathrm{~S}$ & $S$ & $\mathrm{~S}$ & $\mathrm{~S}$ & $\mathrm{~S}$ & $\mathrm{~S}$ \\
\hline $\mathrm{i}$ & Toluene & $S$ & $\mathrm{~S}$ & $\mathrm{~S}$ & $\mathrm{~S}$ & $\mathrm{~S}$ & $\mathrm{~S}$ & $\mathrm{~S}$ \\
\hline j & Methanol & $\mathrm{S}$ & $S$ & $\mathrm{~S}$ & $\mathrm{~S}$ & $\mathrm{~S}$ & $S$ & $\mathrm{~S}$ \\
\hline $\mathrm{k}$ & Ethanol & $\mathrm{S}$ & $S$ & $\mathrm{~S}$ & $\mathrm{~S}$ & $\mathrm{~S}$ & $\mathrm{~S}$ & $\mathrm{~S}$ \\
\hline 1 & 1-butanol & 1 & $\mathrm{P}$ & $\mathrm{S}$ & $\mathrm{S}$ & $\mathrm{S}$ & $\mathrm{P}$ & $\mathrm{P}$ \\
\hline $\mathrm{m}$ & Silicone oil & 1 & I & I & $S$ & $S$ & 1 & I \\
\hline$n$ & o-xylene & $\mathrm{S}$ & $\mathrm{S}$ & $S$ & $\mathrm{~S}$ & $\mathrm{~S}$ & $\mathrm{~S}$ & S \\
\hline 0 & $p$-xylene & $\mathrm{S}$ & $\mathrm{S}$ & $\mathrm{S}$ & $\mathrm{S}$ & $\mathrm{S}$ & $\mathrm{S}$ & $S$ \\
\hline $\mathrm{p}$ & Chlorobenzene & $\mathrm{S}$ & $\mathrm{S}$ & $S$ & $\mathrm{~S}$ & $\mathrm{~S}$ & $S$ & $S$ \\
\hline$q$ & Sesame oil & 1 & OG & I & $\mathrm{P}$ & 1 & I & I \\
\hline$r$ & Mustard oil & 1 & OG & 1 & I & 1 & I & I \\
\hline $\mathrm{s}$ & Eucalyptus oil & i & $\mathrm{P}$ & $\mathrm{S}$ & i & i & i & i \\
\hline $\mathrm{t}$ & Coconut oil & I & OG & I & I & I & I & I \\
\hline $\mathrm{u}$ & Cinnamon oil & 1 & 1 & $\mathrm{~S}$ & I & I & I & I \\
\hline $\mathrm{v}$ & Citriodora oil & I & OG & $\mathrm{S}$ & $\mathrm{S}$ & 1 & 1 & $S$ \\
\hline
\end{tabular}

$\mathrm{I}=$ Insoluble, $\mathrm{S}=$ Soluble, $\mathrm{OG}=$ Opaque Gel, $\mathrm{P}=$ Precipitation

Encouraged by these results, we continued to further optimize the reaction conditions. We then turned our attention to investigate the effect of concentration of a gelator (that is minimum gelation concentration of a gelator) on the magnitude of the gel formation in various organic solvents and oils. Therefore, several gelation experiments have been performed and the resulting data is to be tabulated in Table 2 .

Table 2: Gelation of solvents by using N-(2-aminoethyl)-oleamide at varying concentrations in $w t \% / v$

\begin{tabular}{lccccccc}
\hline Entry & Solvent & 0.4 & 0.8 & 1.2 & 1.4 & 1.6 & 2 \\
\hline i & n-Hexane & $P$ & OG & OG & OG & OG & OG \\
ii & DMSO & $P$ & OG & OG & OG & OG & OG \\
iii & Sesame oil & $P$ & OG & OG & OG & OG & OG \\
iv & Mustard oil & OG & OG & OG & OG & OG & OG \\
v & Coconut oil & OG & OG & OG & OG & OG & OG \\
vi & Citriodora oil & P & P & OG & OG & OG & OG \\
\hline
\end{tabular}

$O G=$ Opaque Gel, $P=$ Precipitation

The closer examination of Table 2 reveals that, the minimum gelation concentration (MGC) for gelator-2 was $0.4 \mathrm{wt} \% / \mathrm{v}$ for gelation of mustard oil (entry, iv) and coconut oil (entry, v), while a precipitate has been formed when gelator-2 is to be added in n-hexane (entry, i), DMSO (entry, ii), sesame oil (entry, iii), and citridira oil (entry, vi). When shifting from $0.4 \mathrm{wt} \% / \mathrm{v}$ to $0.8 \mathrm{wt} \% / \mathrm{v}$ only citriodora oil (entry, vi) gives a precipitate while rest of the solvents and the oils formed gel (entry, $i$ to $v$ ). In the next experimentation the concentration of a gelator-2 has been tuned from 1.2 to $1.6 \mathrm{wt} \% / \mathrm{v}$, and it has been found that it offers gel with every studied solvent system (entry, i to vi).

As we know, the gelation is a reversible process and highly dependent on the temperature (i.e., on heating, gel undergo melting and after cooling, gel is formed again). At the outset of the studies, number of sol-gel transition cycles were performed (almost 10 cycles for each solvent system). To our delight, gel was formed in every cycle.

To ensure the efficacy of a gelator-2, several model gelation reactions were performed with varying concentration of a gelator-2 as well as under different condition of reaction temperature for the gelation of organic solvents like $n$-hexane, DMSO, sesame oil, mustard oil, coconut oil and citriodora oil, and the results are tabulated in the following Table 3.

By comparing the experimental data reported in Table 3, obtained from all the transformations, the stability of a resulting gel was found to be exceptional 
in the case of $n$-hexane (entry, 1). For gelation it is well- known that the hydrogen bonding interactions as well as van der Waal forces play a vital role, and at the outset of our studies we assume that the solutesolvent interactions between $\mathrm{n}$-hexane and gelator-2 yields quite stable gel.

Table 3: Gelation data obtained by using N-(2aminoethyl)-oleamide

\begin{tabular}{ccccc}
\hline Entry & Solvent & $\mathrm{mgc}(\mathrm{wt} \% / \mathrm{v})$ & $\mathrm{Tg}\left({ }^{\circ} \mathrm{C}\right)$ & Stability \\
\hline 1 & $n$-Hexane & 0.8 & $45-46$ & $2 \mathrm{w}$ \\
2 & DMSO & 0.5 & $52-53$ & $10 \mathrm{~m}$ \\
3 & Sesame oil & 0.5 & $65-66$ & $12 \mathrm{~m}$ \\
4 & Mustard oil & 0.4 & $71-72$ & $8 \mathrm{~m}$ \\
5 & Coconut oil & 0.3 & $74-75$ & $8 \mathrm{~m}$ \\
6 & Citriodora oil & 0.9 & $58-59$ & $4 \mathrm{~m}$ \\
\hline \multicolumn{5}{c}{ CONCLUSION }
\end{tabular}

This study suggests that formation of a gel is not only dependent on the nature of a gelator or solvent as well as it is highly dependent on the ease of solute-solute and solute-solvent interactions. In the resent report, we investigated 7 different structurally diverse amides based on oleic acid as a gelator for the gelation of organic solvents as well as edible oils. Clearly, the results presented here are likely to inspire not only other scholars in the field but especially practitioners of the fields beyond chemistry.

\section{ACKNOWLEDGEMENT}

I acknowledge the laboratory services of SAIF, Punjab University, Chandigarh and the support from D. D. N. Bhole college, Bhusawal and Arts, Commerce and Science college, Bodwad for this work.

\section{Conflict of interest}

The author declare that we have no conflict of interest.

\section{REFERENCES}

1. de Rossi, D.; Kajiwara, K.; Osada, Y.; Yamauchi, A. Polymer Gels, Springerlink., 1991, 140-141.

2. de Gennes, P.G. Scaling Concepts in Polymer Physics., 1979.

3. Abdallah, D. J.; Weiss, R. G. Langmuir., 2000, 16, 352.

4. Abdallah, D. J.; Lu, L.; Weiss, R. G. Chem. Mater., 1999, 11, 2907.

5. Lu, L.; Weiss, R. G. Chem. Commun., 1996, 2029.

6. Lin, Y.C.; Kachar, B.; Weiss, R. G. J. Am. Chem. Soc., 1989, 111, 5542.

7. Placin, F.; Desvergne, J.P.; Cansell, F. J. Mater. Chem., 2000, 10, 2147.

8. Kobayashi, H.; Friggeri, A.; Koumoto, K.; Amaike, M.; Shinkai, S. Org. Lett., 2002, 4, 1423.

9. Derossi, D.; Kajiwara, K.; Osada, Y.; Yamauchi. Plenum Press, NewYork., 1991.

10. Bhattacharya, S.; Ghosh, Y. K. Chem. Commun., 2001, 185.

11. Vidal, M. B.; Gil, M. H. J. Bioact. Compat. Polym., 1999, 14, 243.

12. Pozzo, J.L.; Clavier, G. M.; Desvergne, J.P. J. Mater. Chem., 1998, 8, 2575.
13. Loos, M.; Esch, J.; Stokroos, I.; Kellogg, R. M.; Feringa, B. L. J. Am. Chem. Soc., 1997, 119, 12675.

14. Jung, J. H.; Ono, Y.; Shinkai, S. Chem. Eur. J., 2000, 6, 4552.

15. Lortie, F.; Boileau, S.; Bourtiller, L.; Chasseneux, C.; Deme, B.; Ducouret, G.; Jalabert, M.; Laupretre, F.; Terech, P. Langmuir., 2002, 7218-7222.

16. Kobayashi, S.; Hamasaki, N.; Suzuki, M.; Kimura, M.; Shirai, H.; Hanabusa, K. J. Am. Chem. Soc., 2002, 124, 6550.

17. Jung, J. H.; Ono, Y.; Shinkai, S. Angew. Chem., Int. Ed., 2000, 39, 1862.

18. Ajayaghosh, A.; George, S. J. J. Am. Chem. Soc., 2001, 123(21), 5148-5149.

19. Ono, Y.; Nakashima, K.; Sano, M.; Hojo, J.; Shinkai, S. J. Mater. Chem., 2001, 11, 2412.

20. Challis, B.C.; Challis, J. A. Pergamon Press. 1987, 957.

21. Bhilare, S.; Shah, J.; Gaikwad, V.; Gupta, G.R.; Sanghvi, Y.S.; Bhanage, B.M.; Kapdi, A.R. Synthesis., 2019, 51(22), 4239-4248.

22. Gupta, G.R.; Vadagaonkar, K.S.; Lavekar, A.G.; Shah, J.; Kapdi, A.R. Org. Biomol. Chem., 2019, 17, 7596-7631. 
23. Fuhrhop, J.H.; Helfrich, W. Chem. Rev., 1993, 93, 1565.

24. Estroff, L.A.; Hamilton, A.D. Angew. Chem. Int. Ed., 2000, 39, 3447.

25. Cerqueira, M.A.; Fasolin, L.H.; Picone, C.S.F.; Pastrana, L.M.; Cunha, R.L.; Vicente, A.A. Food Research International., 2017, 96, 161170.

26. de Godoi, K.R.R.; Basso, R.C.; Ming, C.C.; de Silva, V.M.; da Cunha, R.L.; Arellano, D.B.; Ribeiro, A.P.B. Food Research International., 2019, 124, 108475.

27. Zeng, C.; Wan, Z.; Xia, H.; Zhao, H.; Guo, S. Food Biophysics., 2020, 15, 452-462.

28. Gupta, G.R.; Nevare, M.R.; Patil, A.M.; Gite,
V.V. Bull. Mater. Sci., 2019, 42(5), 203-214.

29. Gupta, G.R.; Shaikh, V.R.; Kalas, S.; Patil, K. J. Current Physical Chemistry., 2021, 11(1), 18-26.

30. Sarode, C.; Yeole, S.D.; Chaudhari, G.R.; Waghulde, G.P.; Gupta, G.R. Current Physical Chemistry., 2021, 11(1), 27-34.

31. Bhirud, J.D.; Gupta, G.R.; Narkhede, H.P. Russ. J. Org. Chem., 2020, 56(10), 18151822.

32. Girase, T.R.; Patil, K.J.; Kapdi, A.R.; Gupta, G.R.; Chemistry Select., 2020, 5, 42514262.

33. Gupta, G.R.; Shaikh, V.R.; Patil, K. J.; Curr. Phy. Chem., 2018, 8(3), 175-185. 